\title{
Axially variable-length solid element of absolute nodal coordinate formulation
}

\author{
Sun, Jialiang; Tian, Qiang; Hu, Haiyan; Pedersen, Niels L.
}

Published in:

Acta Mechanica Sinica

Link to article, DOI:

$10.1007 / \mathrm{s} 10409-018-0823-7$

Publication date:

2019

Document Version

Peer reviewed version

Link back to DTU Orbit

Citation (APA):

Sun, J., Tian, Q., Hu, H., \& Pedersen, N. L. (2019). Axially variable-length solid element of absolute nodal coordinate formulation. Acta Mechanica Sinica, 35, 653-663 . https://doi.org/10.1007/s10409-018-0823-7

\section{General rights}

Copyright and moral rights for the publications made accessible in the public portal are retained by the authors and/or other copyright owners and it is a condition of accessing publications that users recognise and abide by the legal requirements associated with these rights.

- Users may download and print one copy of any publication from the public portal for the purpose of private study or research.

- You may not further distribute the material or use it for any profit-making activity or commercial gain

- You may freely distribute the URL identifying the publication in the public portal

If you believe that this document breaches copyright please contact us providing details, and we will remove access to the work immediately and investigate your claim. 


\title{
An axially variable-length solid element of absolute nodal coordinate formulation
}

\author{
Jialiang Sun ${ }^{1}$ Qiang Tian ${ }^{2} \cdot$ Haiyan Hu${ }^{1,2, *} \cdot$ Niels L. Pedersen ${ }^{3}$ \\ ${ }^{1}$ State Key Laboratory of Mechanics and Control of Mechanical Structures, College of Aerospace \\ Engineering, Nanjing University of Aeronautics and Astronautics, Nanjing 210016, China \\ ${ }^{2}$ MOE Key Laboratory of Dynamics and Control of Flight Vehicle, School of Aerospace \\ Engineering, Beijing Institute of Technology, Beijing 100081, China \\ ${ }^{3}$ Department of Mechanical Engineering, Solid Mechanics, Technical University of Denmark, \\ Kongens Lyngby 2800, Denmark
}

\begin{abstract}
An axially variable-length solid element with eight nodes is proposed by integrating the arbitrary Lagrangian-Eulerian (ALE) formulation and the absolute nodal coordinate formulation (ANCF). In addition to the nodal positions and slopes of eight nodes, two material coordinates in the axial direction are used as the generalized coordinates. As a consequence, the nodes in the ALE-ANCF are not associated with any specific material points and the axial length of the solid element can be varied over time. The above two material coordinates give rise to a variable mass matrix and an additional inertial force vector. Computationally efficient formulae of the additional inertial forces and elastic forces, as well as their Jacobians, are also derived. The dynamic equation of a flexible multibody system (FMBS) with variable-length bodies is presented. The maximum and minimum lengths of the boundary elements of an FMBS have to be appropriately defined to ensure accuracy and non-singularity when solving the dynamic equation. Three numerical examples of static and dynamic problems are given to validate the variable-length solid elements of ALE-ANCF and show their capability.
\end{abstract}

Keywords Flexible multibody dynamics - Arbitrary Lagrangian-Eulerian formulation - Absolute nodal coordinate formulation · Variable-length solid element

\footnotetext{
* Author to whom all correspondence should be addressed.

E-mail addresses: sunjialiang@nuaa.edu.cn (J. Sun), tianqiang_hust@aliyun.com (Q. Tian), hhyae@nuaa.edu.cn (H. Hu), nlp@mek.dtu.dk (N.L. Pedersen).
} 


\section{Introduction}

Flexible multibody system (FMBS) is an appropriate model for many mechanical systems, such as a vehicle tire, a belt drive, an elastic cable, a helicopter rotor, a high-speed robot and a deployable space structure [1-4]. For the optimization of those systems, their accurate dynamic models have to be established before the design phase. The pioneering work of Shabana [5] in 1996 for the absolute nodal coordinate formulation (ANCF) has been considered a milestone in the development of flexible multibody dynamics to accurately describe an FMBS undergoing both large overall motion and large deformation. As a nonincremental method, the finite elements of ANCF use both nodal positions and slopes, instead of infinitesimal or finite rotations, as the generalized nodal coordinates such that it can lead to zero strain under an arbitrary rigid body motion. Since then, great efforts have been made to propose the finite elements of ANCF for different purposes. The readers can refer to [3] and the references therein for a state-of-the-art review of the beam and plate/shell elements of the ANCF. In recent years, a few solid elements [2, 6-8] of ANCF have been developed for the modeling and topology optimization [1] of a three-dimensional (3D) FMBS, and for fluid dynamics [7].

These finite elements of ANCF, however, cannot describe an FMBS with variable-length bodies, which is a useful model for cranes, reeving systems, cable-driven parallel manipulators, tethered satellites and deployable articulated masts [9-12]. At present, a few formulations are available for the simulation of an FMBS with variable-length bodies, such as the variable-domain finite element (VFE) model based on the floating frame of reference formulation (FFRF) $[13,14]$ and the arbitrary Lagrangian-Eulerian (ALE) description based on the ANCF $[15,16]$. The ALE-ANCF method combines the merits of both ALE formulation and ANCF. It utilizes the position and slope information of a node, which is not tied to any specific material point, but can slide freely in the direction of variable length by means of additional material coordinates $[15,16]$, as the generalized coordinates. The material coordinates in ALE-ANCF give rise to a variable shape function matrix, and consequently, a variable mass matrix and an additional inertial force vector.

Recent years have witnessed the studies on many kinds of variable-length finite elements of ALE-ANCF for various applications. For example, Tang et al. [9] developed an ANCF-based variable-length cable element by combining Lagrangian and Eulerian descriptions and simulated the deployment process of tethered satellite systems. This cable element, however, is only suitable for simple boundary conditions as the boundary elements must be defined in advance [17]. Hong and Ren [15] and Hong et al. [18] developed a variable-length beam element of ALE-ANCF by introducing two material coordinates of the two nodes as the generalized coordinates. They simulated the FMBS involving complex boundary conditions, such as the sliding joint on a cable and a tubular cantilever conveying fluid. However, Hong and Ren [15] and Hong et al. [18] neglected the torsion of the proposed beam element. Du 
et al. [11] developed a variable-length cable element by simply using node positions as the generalized coordinates and neglecting the bending and torsion deformations. They used the cable element to efficiently simulate the dynamics of cable-driven parallel manipulators. Escalona [10] developed a variable-length cable element by accounting for the axial-torsion of the element and simulated classic reeving systems. He used two material coordinates and two rotation angles of the cross-section as the generalized coordinates, but did not include the nodal slopes in the absolute nodal coordinates. Hence, the two cable elements by Du et al. [11] and Escalona [10] are not the finite elements of ANCF according to the definition by Shabana [19]. Yang et al. [17] generalized these variable-length beam elements of ANCF aforementioned and proposed a novel variable-length beam element that uses positions and slopes of two nodes, two material coordinates and two rotation angles of the cross-section as the generalized coordinates. This beam element, therefore, enables one to model flexible variable-length beam structures, for which the torsional effect is not negligible. For the plate problem involving variable length, Hyldahl et al. [16] initially proposed the variable-length thin plate element of ANCF by introducing four material coordinates to facilitate that both the length and width of the plate element can vary. Sun et al. [20] performed the topology optimization of planar variable-length structures in an FMBS modeled via the variable-length thin plate element of ALE-ANCF.

This study focuses on the development and verification of an axially variable-length solid element with eight nodes of ANCF and presents the formulae of the elastic forces, additional inertial forces as well as their Jacobians. The solid element of concern can vary its length in the axial direction so as to describe a $3 \mathrm{D}$ FMBS with variable-length bodies. Compared with the solid element of ANCF [6], the solid element with variable length uses two additional material coordinates in the axial direction as the generalized coordinates. As the absolute nodal coordinates, i.e., the positions and slopes of nodes, still keep as the nodal coordinates, the solid element is capable of describing a $3 \mathrm{D}$ FMBS subject to both large overall motion and large deformation.

\section{Implementation of a solid element of ALE-ANCF}

\subsection{Basic descriptions}

The solid element is assumed to be able to vary its length in one direction (z-direction in this study) by introducing two material coordinates. As shown in Fig. 1, the generalized coordinates of the solid element of ALE-ANCF can be expressed as

$$
\mathbf{q}=\left[\begin{array}{ll}
\mathbf{q}_{e}^{\mathrm{T}} & \mathbf{q}_{l}^{\mathrm{T}}
\end{array}\right]^{\mathrm{T}}
$$

where $\quad \mathbf{q}_{e}=\left[\begin{array}{lllllllll}\mathbf{r}_{1}^{\mathrm{T}} & \mathbf{r}_{1, x}^{\mathrm{T}} & \mathbf{r}_{1, y}^{\mathrm{T}} & \mathbf{r}_{1, z}^{\mathrm{T}} & \cdots & \mathbf{r}_{8}^{\mathrm{T}} & \mathbf{r}_{8, x}^{\mathrm{T}} & \mathbf{r}_{8, y}^{\mathrm{T}} & \mathbf{r}_{8, z}^{\mathrm{T}}\end{array}\right]^{\mathrm{T}}$ is the 96 traditional generalized coordinates of ANCF with $\mathbf{r}_{i, x}=\partial \mathbf{r}_{i} / \partial x, \mathbf{r}_{i, y}=\partial \mathbf{r}_{i} / \partial y$ and $\mathbf{r}_{i, z}=\partial \mathbf{r}_{i} / \partial z$ 
denoting the three slopes of node i. $\mathbf{q}_{l}=\left[\begin{array}{ll}h_{1} & h_{2}\end{array}\right]^{\mathrm{T}}$ is the 2 material coordinates measured along the $z$-direction of the undeformed flexible body from the head face. The head face is determined as one of the cross-sections of the undeformed flexible body, in which the material coordinate is constant For example, it is zero in this study. For one solid element of ALE-ANCF in Fig. 1, hence, there are 98 generalized coordinates.

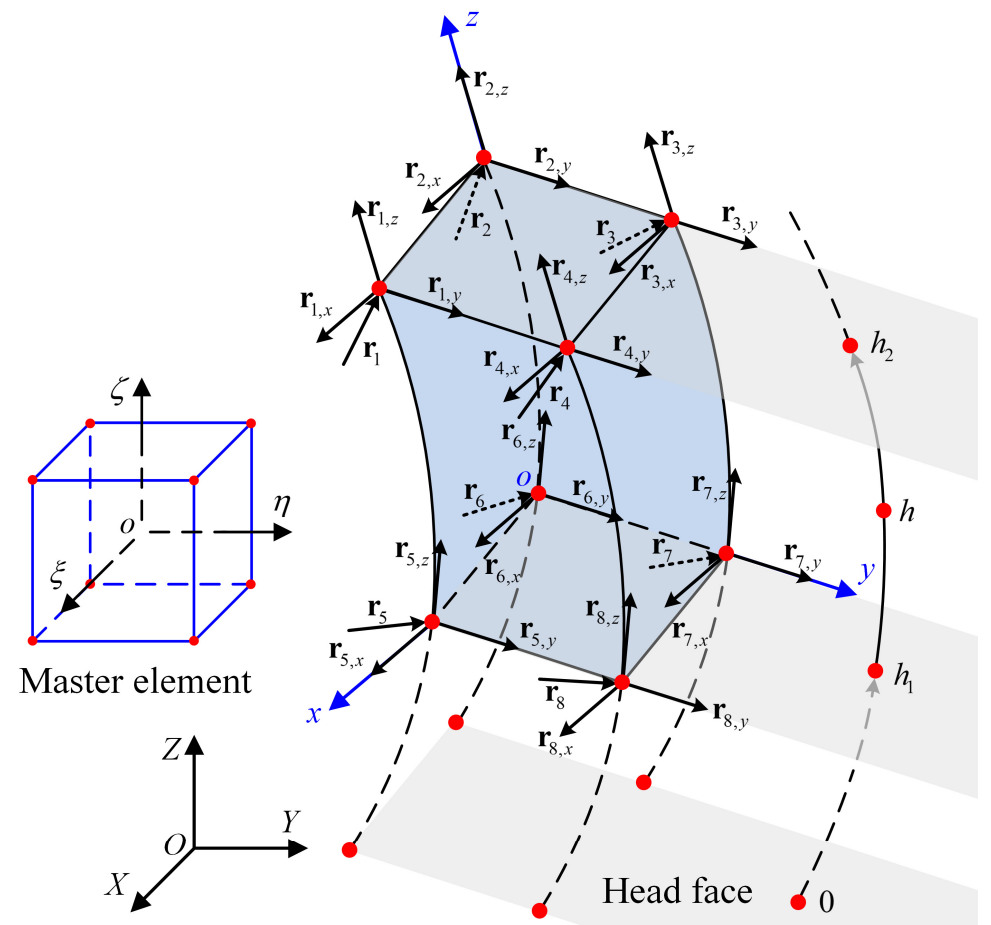

Fig. 1 A deformed solid element with eight nodes of ALE-ANCF

In the description of Eq. (1), the element nodes are no longer fixed to any material points in the flexible variable-length body and can slide freely in the axial direction (z-direction) under the control of $h_{1}$ and $h_{2}$. If $\dot{h}_{2}-\dot{h}_{1}>0$, the undeformed length of the solid element increases. Otherwise, it decreases or keeps constant. If $\dot{h}_{2}=\dot{h}_{1} \neq 0$, the undeformed length of the solid element is constant although the solid element is sliding along the flexible body. If $\dot{h}_{2}=\dot{h}_{1}=0$, the element nodes are fixed to specific material points and the solid element of ALE-ANCF becomes the solid element of ANCF. If this is the case, all the properties of a solid element of ANCF hold true for a solid element of ALE-ANCF.

In the global frame of coordinates $O-X Y Z$, the position vector of an arbitrary point on the element can be determined as

$$
\mathbf{r}=\mathbf{N}_{e} \mathbf{q}_{e},
$$

where $\quad \mathbf{N}_{e}=\left[\begin{array}{lllllllll}N_{1,0} \mathbf{I} & N_{1,1} \mathbf{I} & N_{1,2} \mathbf{I} & N_{1,3} \mathbf{I} & \cdots & N_{8,0} \mathbf{I} & N_{8,1} \mathbf{I} & N_{8,2} \mathbf{I} & N_{8,3} \mathbf{I}\end{array}\right]$ is the interpolation function matrix with $\mathbf{I}$ denoting the $3 \times 3$ identity matrix. The entries of $\mathbf{N}_{e}$ are defined as follows 


$$
\begin{aligned}
& N_{i, 0}=\frac{1}{16}(1 \pm \xi)(1 \pm \eta)(1 \pm \zeta)\left(2 \pm \xi \pm \eta \pm \zeta-\xi^{2}-\eta^{2}-\zeta^{2}\right), \\
& N_{i, 1}= \pm \frac{1}{32} a\left(\xi^{2}-1\right)(1 \pm \xi)(1 \pm \eta)(1 \pm \zeta), \\
& N_{i, 2}= \pm \frac{1}{32} b\left(\eta^{2}-1\right)(1 \pm \xi)(1 \pm \eta)(1 \pm \zeta), \\
& N_{i, 3}= \pm \frac{1}{32} c\left(\zeta^{2}-1\right)(1 \pm \xi)(1 \pm \eta)(1 \pm \zeta) .
\end{aligned}
$$

where $i=1,2, \cdots, 8, a, b$ and $c=h_{2}-h_{1}$ are the lengths of the undeformed solid element in $x$-, $y$ - and $z$-directions, respectively. $\xi=\frac{x-a / 2}{a / 2}(0 \leq x \leq a), \quad \eta=\frac{y-b / 2}{b / 2}$ $(0 \leq y \leq b)$, and $\zeta=\frac{z-c / 2}{c / 2}(0 \leq z \leq c)$ are the isoparametric coordinates of the corresponding master element, as shown in Fig. 1. As the frame $o-x y z$ is the element-fixed local frame of coordinates, the local coordinate $z$ and the material coordinate $h$ yield the relationship $z=h-h_{1}$. It is worth mentioning that the variable $h_{1}$ depends on time $t$ only. Then, the isoparametric coordinate $\zeta$ can be rewritten as $\zeta=\frac{2 h-h_{1}-h_{2}}{h_{2}-h_{1}} \quad\left(h_{1} \leq h \leq h_{2}\right)$. The more detailed expressions of Eq. (3) can be seen in [21].

According to Eq. (2), the vectors of velocity and acceleration of an arbitrary point in the solid element can be derived as

$$
\begin{aligned}
& \dot{\mathbf{r}}=\mathbf{N}_{e} \dot{\mathbf{q}}_{e}+\left(\mathbf{N}_{e, h_{1}} \dot{h}_{1}+\mathbf{N}_{e, h_{2}} \dot{h}_{2}\right) \mathbf{q}_{e}, \\
& \ddot{\mathbf{r}}=\mathbf{N}_{e} \ddot{\mathbf{q}}_{e}+\left(\mathbf{N}_{e, h_{1}} \ddot{h}_{1}+\mathbf{N}_{e, h_{2}} \ddot{h}_{2}\right) \mathbf{q}_{e}+\mathbf{a}_{h},
\end{aligned}
$$

with

$$
\mathbf{a}_{h}=2\left(\mathbf{N}_{e, h_{1}} \dot{h}_{1}+\mathbf{N}_{e, h_{2}} \dot{h}_{2}\right) \dot{\mathbf{q}}_{e}+\left(\mathbf{N}_{e, h_{1} h_{1}} \dot{h}_{1}^{2}+2 \mathbf{N}_{e, h_{1} h_{2}} \dot{h}_{1} \dot{h}_{2}+\mathbf{N}_{e, h_{2} h_{2}} \dot{h}_{2}^{2}\right) \mathbf{q}_{e},
$$

where $\mathbf{N}_{e, h_{1}}$ denotes $\partial \mathbf{N}_{e} / \partial h_{1}$ and $\mathbf{N}_{e, h_{1} h_{1}}$ denotes $\partial^{2} \mathbf{N}_{e} / \partial h_{1} \partial h_{1}$. For further discussion, the shape function matrix is defined as $\mathbf{N}=\left[\begin{array}{llll}\mathbf{N}_{e} & \mathbf{N}_{e, h_{1}} \mathbf{q}_{e} & \mathbf{N}_{e, h_{2}} \mathbf{q}_{e}\end{array}\right]$, and Eq. (4) can be rewritten as

$$
\dot{\mathbf{r}}=\mathbf{N} \dot{\mathbf{q}}, \ddot{\mathbf{r}}=\mathbf{N} \ddot{\mathbf{q}}+\mathbf{a}_{h} .
$$

As the solid element of ALE-ANCF has a variable mass due to the variable length, the more general d'Alembert's principle [22] is used to establish the dynamic equation of an unconstrainted element in the following way

$$
\mathbf{M}_{e l e} \ddot{\mathbf{q}}+\mathbf{F}^{a}+\mathbf{F}^{e}=\mathbf{Q},
$$

where $\mathbf{M}_{\text {ele }}$ the time-dependent mass matrix of the element, $\mathbf{F}^{e}$ is the vector of elastic forces, $\mathbf{F}^{a}$ is the vector of additional inertial forces associated with $\mathbf{a}_{h}$ and 
$\mathbf{Q}$ is the vector of generalized external forces, including gravity, actuating forces and damping forces. They can be derived as follows

$$
\begin{aligned}
& \mathbf{M}_{e l e}=\int_{V_{0}} \rho \mathbf{N}^{\mathrm{T}} \mathbf{N} \mathrm{d} V=\frac{1}{8} a b c \int_{-1}^{1} \int_{-1}^{1} \int_{-1}^{1} \rho \mathbf{N}^{\mathrm{T}} \mathbf{N} \mathrm{d} \xi \mathrm{d} \eta \mathrm{d} \zeta, \\
& \mathbf{F}^{e}=\int_{V_{0}}\left(\frac{\partial \boldsymbol{\varepsilon}}{\partial \mathbf{q}}\right)^{\mathrm{T}} \mathbf{E} \boldsymbol{\varepsilon} \mathrm{d} V=\frac{1}{8} a b c \int_{-1}^{1} \int_{-1}^{1} \int_{-1}^{1}\left(\frac{\partial \boldsymbol{\varepsilon}}{\partial \mathbf{q}}\right)^{\mathrm{T}} \mathbf{E} \boldsymbol{\varepsilon} \mathrm{d} \xi \mathrm{d} \eta \mathrm{d} \zeta, \\
& \mathbf{F}^{a}=\int_{V_{0}} \rho \mathbf{N}^{\mathrm{T}} \ddot{\mathbf{r}}_{p} \mathrm{~d} V=\frac{1}{8} a b c \int_{-1}^{1} \int_{-1}^{1} \int_{-1}^{1} \rho \mathbf{N}^{\mathrm{T}} \mathbf{a}_{h} \mathrm{~d} \xi \mathrm{d} \eta \mathrm{d} \zeta, \\
& \mathbf{Q}=\int_{V_{0}} \mathbf{N}^{\mathrm{T}} \mathbf{f} \mathrm{d} V=\frac{1}{8} a b c \int_{-1}^{1} \int_{-1}^{1} \int_{-1}^{1} \mathbf{N}^{\mathrm{T}} \mathbf{f} \mathrm{d} \xi \mathrm{d} \eta \mathrm{d} \zeta,
\end{aligned}
$$

where $V_{0}$ is the initial volume of the solid element, $\rho$ is the density, $\boldsymbol{\varepsilon}$ is the Green strain tensor, $\mathbf{f}$ is the vector of external body forces, $\mathbf{E}$ is the elastic coefficient matrix with $E$ denoting Young's modulus and $v$ denoting Poisson's ratio as follows

$$
\mathbf{E}=\frac{E}{(1+v)(1-2 v)}\left[\begin{array}{cccccc}
1-v & v & v & 0 & 0 & 0 \\
v & 1-v & v & 0 & 0 & 0 \\
v & v & 1-v & 0 & 0 & 0 \\
0 & 0 & 0 & \frac{1-2 v}{2} & 0 & 0 \\
0 & 0 & 0 & 0 & \frac{1-2 v}{2} & 0 \\
0 & 0 & 0 & 0 & 0 & \frac{1-2 v}{2}
\end{array}\right] .
$$

Different from the conventional ANCF element [1], the solid element of ALE-ANCF includes an additional term $\mathbf{F}^{a}$ associated with $\mathbf{a}_{h}$ in the dynamic equation (7). The term $\mathbf{F}^{a}$ exists for the reason that there is a mass flowing in or out of the element. If the eight nodes of the solid element are fixed to the material points, that is, $\dot{h}_{2}=\dot{h}_{1}=0, \mathbf{a}_{h}$ will be equal to zero according to Eq. (5). As a consequence, the vector of additional inertial forces $\mathbf{F}^{a}$ in Eq. (10) will vanish. In practice, if the deployment or retraction law of the flexible body is known, $\dot{h}_{1}$ and $\dot{h}_{2}$ can be easily determined. If the law is unknown, $\dot{h}_{1}$ and $\dot{h}_{2}$ should be computed from the dynamic simulation.

\subsection{Formulations of elastic forces and corresponding Jacobian}

The strain tensor $\boldsymbol{\varepsilon}$ in Eq. (9) can be expressed as 


$$
\boldsymbol{\varepsilon}=\left[\begin{array}{c}
\left(\mathbf{r}_{, x}^{\mathrm{T}} \mathbf{r}_{, x}-1\right) / 2 \\
\left(\mathbf{r}_{, y}^{\mathrm{T}} \mathbf{r}_{, y}-1\right) / 2 \\
\left(\mathbf{r}_{, h}^{\mathrm{T}} \mathbf{r}_{, h}-1\right) / 2 \\
\mathbf{r}_{, x}^{\mathrm{T}} \mathbf{r}_{, y} \\
\mathbf{r}_{, y}^{\mathrm{T}} \mathbf{r}_{, h} \\
\mathbf{r}_{, h}^{\mathrm{T}} \mathbf{r}_{, x}
\end{array}\right]=\left[\begin{array}{c}
\left((\mathbf{A})_{k m} q_{k} q_{m}-1\right) / 2 \\
\left((\mathbf{B})_{k m} q_{k} q_{m}-1\right) / 2 \\
\left((\mathbf{C})_{k m} q_{k} q_{m}-1\right) / 2 \\
(\mathbf{D})_{k m} q_{k} q_{m} \\
(\mathbf{G})_{k m} q_{k} q_{m} \\
(\mathbf{H})_{k m} q_{k} q_{m}
\end{array}\right]
$$

where $k, m=1,2, \cdots, 96, \quad \mathbf{A}=\mathbf{N}_{e, x}^{\mathrm{T}} \mathbf{N}_{e, x}, \quad \mathbf{B}=\mathbf{N}_{e, y}^{\mathrm{T}} \mathbf{N}_{e, y}, \quad \mathbf{C}=\mathbf{N}_{e, h}^{\mathrm{T}} \mathbf{N}_{e, h}, \quad \mathbf{D}=\mathbf{N}_{e, x}^{\mathrm{T}} \mathbf{N}_{e, y}$, $\mathbf{G}=\mathbf{N}_{e, y}^{\mathrm{T}} \mathbf{N}_{e, h}$, and $\mathbf{H}=\mathbf{N}_{e, h}^{\mathrm{T}} \mathbf{N}_{e, x}$.

The entries in the vector $\mathbf{F}^{e}$ of elastic forces in Eq. (9) can be efficiently computed as follows

$$
F_{i}^{e}= \begin{cases}\frac{1}{2}\left(\left(\mathbf{K}_{1}\right)_{k m n i}+\left(\mathbf{K}_{2}\right)_{k m n i}+\left(\mathbf{K}_{3}\right)_{k m n i}\right) q_{k} q_{m} q_{n} & \\ -\frac{1}{2}\left(\left(\mathbf{K}_{4}\right)_{n i}+\left(\mathbf{K}_{5}\right)\right. & ) \\ n n i & \\ \frac{1}{4}\left(\left(\mathbf{K}_{6}\right)_{k m n l i}+\left(\mathbf{K}_{7}\right)_{k m n l i}+\left(\mathbf{K}_{8}\right)_{k m n l i}\right) q_{k} q_{m} q_{n} q_{l} & \\ -\frac{1}{4}\left(\left(\mathbf{K}_{9}\right)_{n l i}+\left(\mathbf{K}_{10}\right)_{n l i}\right) q_{n} q_{l}, & (i=97,98)\end{cases}
$$

where the subscripts $k, m, n, l=1,2, \cdots, 96$ and the detailed expressions of $\left(\mathbf{K}_{1}\right)_{k m n i}$, $\left(\mathbf{K}_{2}\right)_{k m n i},\left(\mathbf{K}_{3}\right)_{k m n i},\left(\mathbf{K}_{4}\right)_{n i},\left(\mathbf{K}_{5}\right)_{n i},\left(\mathbf{K}_{6}\right)_{k m n l i},\left(\mathbf{K}_{7}\right)_{k m n l i},\left(\mathbf{K}_{8}\right)_{k m n l i},\left(\mathbf{K}_{9}\right)_{n l i}$ and $\left(\mathbf{K}_{10}\right)_{n l i}$ are listed in the "Appendix".

According to Eq. (14), the entries in the Jacobian of the elastic forces can be expressed as follows:

when $i=1,2, \cdots, 96$ and $j=1,2, \cdots, 96$,

$$
\begin{aligned}
\frac{\partial F_{i}^{e}}{\partial q_{j}}= & \frac{1}{2}\left(\left(\mathbf{K}_{1}\right)_{k m j i}+2\left(\mathbf{K}_{1}\right)_{j k m i}+\left(\mathbf{K}_{2}\right)_{k m j i}+2\left(\mathbf{K}_{2}\right)_{j k m i}\right. \\
& \left.+\left(\mathbf{K}_{3}\right)_{k m j i}+\left(\mathbf{K}_{3}\right)_{k j m i}+\left(\mathbf{K}_{3}\right)_{j k m i}\right) q_{k} q_{m} \\
& -\frac{1}{2}\left(\left(\mathbf{K}_{4}\right)_{j i}+\left(\mathbf{K}_{5}\right)_{j i}\right),
\end{aligned}
$$

when $i=1,2, \cdots, 96$ and $j=97,98$,

$$
\begin{aligned}
\frac{\partial F_{i}^{e}}{\partial q_{j}}= & \frac{1}{2}\left(\left(\mathbf{K}_{1}\right)_{k m n i, q_{j}}+\left(\mathbf{K}_{2}\right)_{k m n i, q_{j}}+\left(\mathbf{K}_{3}\right)_{k m n i, q_{j}}\right) q_{k} q_{m} q_{n} \\
& -\frac{1}{2}\left(\left(\mathbf{K}_{4}\right)_{n i, q_{j}}+\left(\mathbf{K}_{5}\right)_{n i, q_{j}}\right) q_{n},
\end{aligned}
$$

when $i=97,98$ and $j=1,2, \cdots, 96$, 


$$
\begin{aligned}
\frac{\partial F_{i}^{e}}{\partial q_{j}}= & \frac{1}{4}\left(2\left(\mathbf{K}_{6}\right)_{k m n j i}+2\left(\mathbf{K}_{6}\right)_{k j m n i}+2\left(\mathbf{K}_{7}\right)_{k m n j i}+2\left(\mathbf{K}_{7}\right)_{k j m n i}\right. \\
& \left.+\left(\mathbf{K}_{8}\right)_{k m n j i}+\left(\mathbf{K}_{8}\right)_{k m j n i}+\left(\mathbf{K}_{8}\right)_{k j m n i}+\left(\mathbf{K}_{8}\right)_{j k m n i}\right) q_{k} q_{m} q_{n} \\
& -\frac{1}{4}\left(2\left(\mathbf{K}_{9}\right)_{n j i}+2\left(\mathbf{K}_{10}\right)_{n j i}\right) q_{n},
\end{aligned}
$$

when $i=97,98$ and $j=97,98$,

$$
\begin{aligned}
\frac{\partial F_{i}^{e}}{\partial q_{j}}= & \frac{1}{4}\left(\left(\mathbf{K}_{6}\right)_{k m n l i, q_{j}}+\left(\mathbf{K}_{7}\right)_{k m n l i, q_{j}}+\left(\mathbf{K}_{8}\right)_{k m n l i, q_{j}}\right) q_{k} q_{m} q_{n} q_{l} \\
& -\frac{1}{4}\left(\left(\mathbf{K}_{9}\right)_{n l i, q_{j}}+\left(\mathbf{K}_{10}\right)_{n l i, q_{j}}\right) q_{n} q_{l} .
\end{aligned}
$$

In Eq. (16), $\left(\mathbf{K}_{1}\right)_{k m n i, q_{j}}$ denotes the partial derivatives of $\left(\mathbf{K}_{1}\right)_{k m n i}$ with respect to the material coordinates $q_{j}(j=97,98)$. According to Eq. (A.1), $\left(\mathbf{K}_{1}\right)_{k m n i, q_{j}}$ can be computed as follows

$$
\begin{aligned}
\left(\mathbf{K}_{1}\right)_{k m n i, q_{j}}=\int_{V_{0}}(\mathbf{E})_{11} & (\mathbf{A})_{k m}\left(\mathbf{A}_{, q_{j}}\right)_{n i}+\left(\mathbf{A}_{, q_{j}}\right)_{k m}(\mathbf{A})_{n i}+(\mathbf{B})_{k m}\left(\mathbf{B}_{, q_{j}}\right)_{n i} \\
& \left.+\left(\mathbf{B}_{, q_{j}}\right)_{k m}(\mathbf{B})_{n i}+(\mathbf{C})_{k m}\left(\mathbf{C}_{, q_{j}}\right)_{n i}+\left(\mathbf{C}_{, q_{j}}\right)_{k m}(\mathbf{C})_{n i}\right) \mathrm{d} V,
\end{aligned}
$$

where the subscripts $k, m, n, i=1,2, \cdots, 96$ and $j=97,98$. It is the same case for $\left(\mathbf{K}_{2}\right)_{k m n i, q_{j}}, \ldots,\left(\mathbf{K}_{9}\right)_{n l i, q_{j}}$ and $\left(\mathbf{K}_{10}\right)_{n l i, q_{j}}$ in Eqs. (16) and (18).

\subsection{Formulations of additional inertial forces and corresponding Jacobian}

According to Eq. (10), the entries in the vector $\mathbf{F}^{a}$ of additional inertial forces can be expressed as follows

$$
F_{i}^{a}=\left\{\begin{array}{l}
\left(\dot{h}_{1}\left(\mathbf{K}_{11}\right)_{i k}+\dot{h}_{2}\left(\mathbf{K}_{12}\right)_{i k}\right) \dot{q}_{k} \\
+\left(\dot{h}_{1}^{2}\left(\mathbf{K}_{13}\right)_{i k}+\dot{h}_{2}^{2}\left(\mathbf{K}_{14}\right)_{i k}+\dot{h}_{1} \dot{h}_{2}\left(\mathbf{K}_{15}\right)_{i k}\right) q_{k}, \\
\left(\dot{h}_{1}\left(\mathbf{K}_{16}\right)_{i l k}+\dot{h}_{2}\left(\mathbf{K}_{17}\right)_{i l k}\right) q_{l} \dot{q}_{k} \\
+\left(\dot{h}_{1}^{2}\left(\mathbf{K}_{18}\right)_{i l k}+\dot{h}_{2}^{2}\left(\mathbf{K}_{19}\right)_{i l k}+\dot{h}_{1} \dot{h}_{2}\left(\mathbf{K}_{20}\right)_{i l k}\right) q_{l} q_{k},
\end{array} \quad(i=1,2, \cdots, 96)\right.
$$

where the subscripts $k, l=1,2, \ldots, 96$ and the detailed expressions of $\left(\mathbf{K}_{11}\right)_{i k}$, $\left(\mathbf{K}_{12}\right)_{i k}, \quad\left(\mathbf{K}_{13}\right)_{i k}, \quad\left(\mathbf{K}_{14}\right)_{i k}, \quad\left(\mathbf{K}_{15}\right)_{i k},\left(\mathbf{K}_{16}\right)_{i l k}, \quad\left(\mathbf{K}_{17}\right)_{i l k}, \quad\left(\mathbf{K}_{18}\right)_{i l k}, \quad\left(\mathbf{K}_{19}\right)_{i l k}$ and $\left(\mathbf{K}_{20}\right)_{i l k}$ are listed in the "Appendix".

The entries in the Jacobian of the additional inertial forces can be expressed as follows:

when $i=1,2, \cdots, 96$ and $j=1,2, \cdots, 96$, 


$$
\frac{\partial F_{i}^{a}}{\partial q_{j}}=\dot{h}_{1}^{2}\left(\mathbf{K}_{13}\right)_{i j}+\dot{h}_{2}^{2}\left(\mathbf{K}_{14}\right)_{i j}+\dot{h}_{1} \dot{h}_{2}\left(\mathbf{K}_{15}\right)_{i j},
$$

when $i=1,2, \cdots, 96$ and $j=97,98$,

$$
\begin{aligned}
\frac{\partial F_{i}^{a}}{\partial q_{j}}= & \left(\dot{h}_{1}\left(\mathbf{K}_{11}\right)_{i k, q_{j}}+\dot{h}_{2}\left(\mathbf{K}_{12}\right)_{i k, q_{j}}\right) \dot{q}_{k} \\
& +\left(\dot{h}_{1}^{2}\left(\mathbf{K}_{13}\right)_{i k, q_{j}}+\dot{h}_{2}^{2}\left(\mathbf{K}_{14}\right)_{i k, q_{j}}+\dot{h}_{1} \dot{h}_{2}\left(\mathbf{K}_{15}\right)_{i k, q_{j}}\right) q_{k},
\end{aligned}
$$

when $i=97,98$ and $j=1,2, \cdots, 96$,

$$
\begin{aligned}
\frac{\partial F_{i}^{a}}{\partial q_{j}}= & \left(\dot{h}_{1}\left(\mathbf{K}_{16}\right)_{i j k}+\dot{h}_{2}\left(\mathbf{K}_{17}\right)_{i j k}\right) \dot{q}_{k} \\
& +\left(\dot{h}_{1}^{2}\left(\mathbf{K}_{18}\right)_{i j k}+\dot{h}_{1}^{2}\left(\mathbf{K}_{18}\right)_{i k j}+\dot{h}_{2}^{2}\left(\mathbf{K}_{19}\right)_{i j k}\right. \\
& \left.+\dot{h}_{2}^{2}\left(\mathbf{K}_{19}\right)_{i k j}+\dot{h}_{1} \dot{h}_{2}\left(\mathbf{K}_{20}\right)_{i j k}+\dot{h}_{1} \dot{h}_{2}\left(\mathbf{K}_{20}\right)_{i k j}\right) q_{k},
\end{aligned}
$$

when $i=97,98$ and $j=97,98$,

$$
\begin{aligned}
\frac{\partial F_{i}^{a}}{\partial q_{j}}= & \left(\dot{h}_{1}\left(\mathbf{K}_{16}\right)_{i l k, q_{j}}+\dot{h}_{2}\left(\mathbf{K}_{17}\right)_{i l k, q_{j}}\right) q_{l} \dot{q}_{k} \\
& +\left(\dot{h}_{1}^{2}\left(\mathbf{K}_{18}\right)_{i l k, q_{j}}+\dot{h}_{2}^{2}\left(\mathbf{K}_{19}\right)_{i l k, q_{j}}+\dot{h}_{1} \dot{h}_{2}\left(\mathbf{K}_{20}\right)_{i l k, q_{j}}\right) q_{l} q_{k} .
\end{aligned}
$$

In Eq. (22), $\left(\mathbf{K}_{11}\right)_{i k, q_{j}}$ denotes the partial derivatives of $\left(\mathbf{K}_{11}\right)_{i k}$ with respect to the material coordinates $q_{j}(j=97,98)$. According to Eq. (A.2) can be computed as

$$
\left(\mathbf{K}_{11}\right)_{i k, q_{j}}=2 \rho \int_{V_{0}}\left(\left(\mathbf{N}_{e}\right)_{d i}\left(\mathbf{N}_{e, h_{1} q_{j}}\right)_{d k}+\left(\mathbf{N}_{e, q_{j}}\right)_{d i}\left(\mathbf{N}_{e, h_{1}}\right)_{d k}\right) \mathrm{d} V,
$$

where the subscripts $i, k=1,2, \ldots, 96, j=97,98$ and $d=1,2,3$. It is the same case for $\left(\mathbf{K}_{12}\right)_{i k, q_{j}}, \ldots,\left(\mathbf{K}_{19}\right)_{i l k, q_{j}}$ and $\left(\mathbf{K}_{20}\right)_{i l k, q_{j}}$ in Eqs. (22) and (24).

\section{Dynamic equation of an FMBS with variable length}

The dynamic equation of an FMBS with variable-length bodies modeled by the ALE-ANCF yields [9, 16]

$$
\left\{\begin{array}{l}
\mathbf{M}(t) \ddot{\mathbf{q}}_{D}+\mathbf{F}^{e}+\mathbf{F}^{a}+\boldsymbol{\Phi}_{, \mathbf{q}_{D}}^{\mathrm{T}} \boldsymbol{\lambda}=\mathbf{Q} \\
\mathbf{\Phi}\left(\mathbf{q}_{D}, t\right)=\mathbf{0}
\end{array}\right.
$$

where $\mathbf{M}$ is the time-dependent mass matrix of the system, $\mathbf{q}_{D}$ is the vector of generalized coordinates of the system, $\mathbf{F}^{e}, \mathbf{F}^{a}, \boldsymbol{\Phi}_{, \mathbf{q}_{D}}^{\mathrm{T}} \boldsymbol{\lambda}$ and $\mathbf{Q}$ represent the vectors of elastic forces of the system, the additional inertial forces of the system, the constraint forces and the generalized external forces, respectively. $\boldsymbol{\Phi}\left(\mathbf{q}_{D}, t\right)=\mathbf{0}$ is the kinematic constraints and $\boldsymbol{\Phi}_{, \mathbf{q}_{D}}$ denotes the Jacobian of $\boldsymbol{\Phi} . \lambda$ is the vector of Lagrange multiples. 

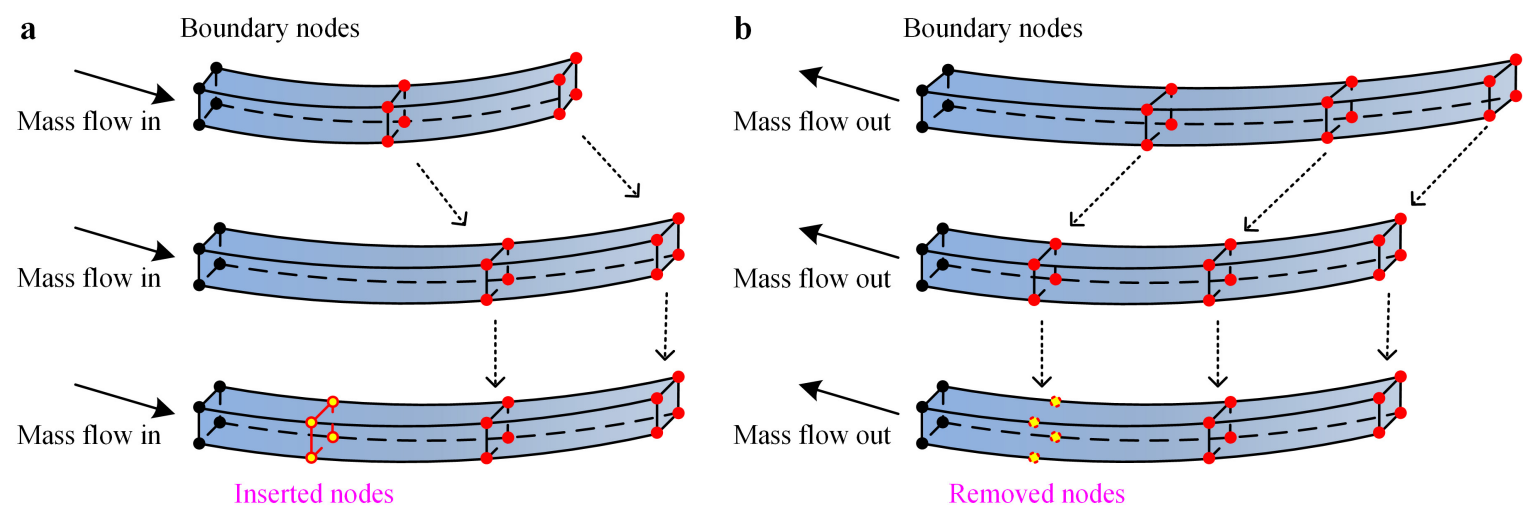

Fig. 2 Illustration of boundary element treatment. a Inserting nodes. b Removing nodes

In this study, the generalized- $\alpha$ method [23-25] is employed to numerically solve the index-3 differential algebraic equations (DAEs) shown in Eq. (26). During the solving procedure, however, one should pay special attention to the lengths of the boundary elements so as to avoid excessively long or excessively short lengths. This is due to the fact that excessively long lengths of boundary elements can reduce the accuracy of the computational result, while the excessively short case can result in the singularity of the stiffness matrix. As illustrated in Fig. 2, when the length of a boundary element is longer than a given maximum length, four new nodes are inserted into the boundary element to divide it into two elements. On the contrary, when the length of the boundary element is shorter than a given minimum length, the two boundary elements merge into one.

\section{Numerical examples}

\subsection{A cantilever beam bent to a helical form}

This is a classic static problem firstly studied by Ibrahimbegovic [26] and also by many others [27-29] to validate the beam elements undergoing large deformations. In this study, this static problem is also employed to validate the accuracy of the variable-length solid element of ALE-ANCF undergoing large deformation in comparison with the geometrically exact three-dimensional beam [29].

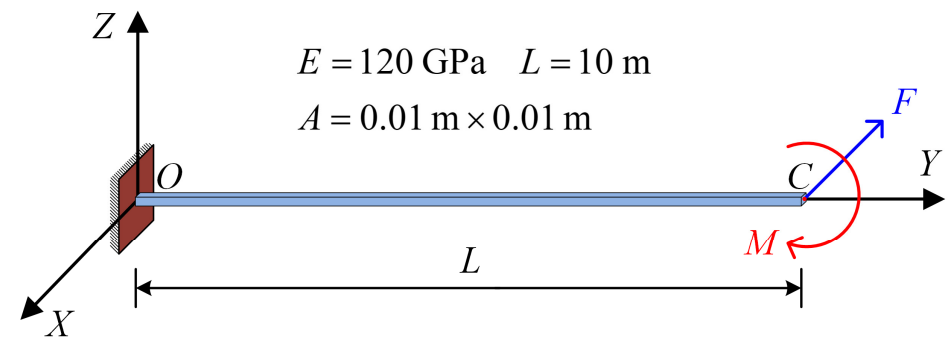

Fig. 3 A schematic diagram of the undeformed beam and the loading case 

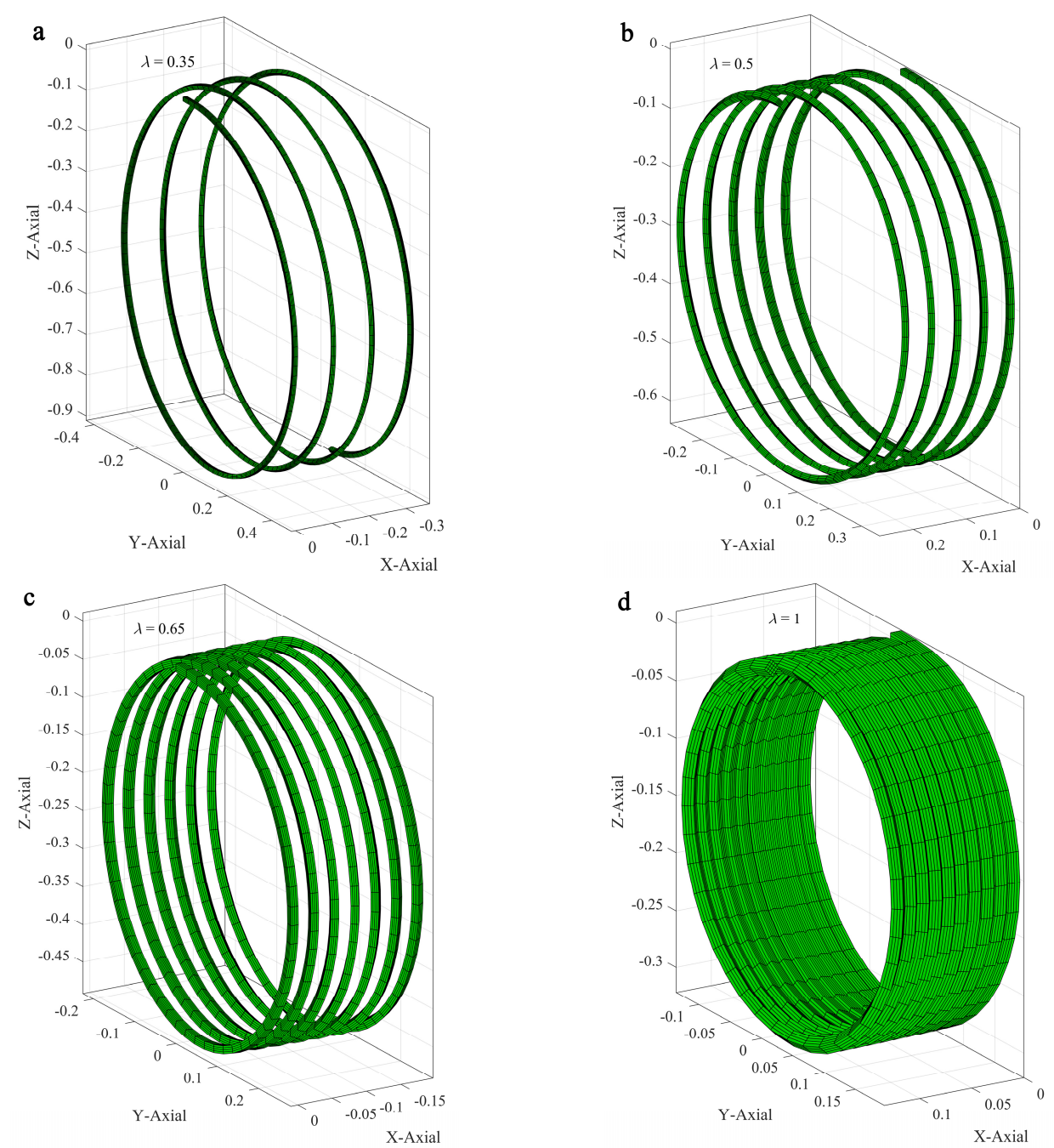

Fig. 4 Deformed configurations of the beam at four loading cases. a $\lambda=0.35$. $\mathbf{b} \lambda=0.5$. $\mathbf{c} \lambda=0.65$. $\mathbf{d} \lambda=1$

As shown in Fig. 3, a slender cantilever beam is subjected to a concentrated moment $M$ and an out-of-plane concentrated force $F$ at its free end. The beam has a length of $10 \mathrm{~m}$ and a square cross-section of $0.01 \mathrm{~m} \times 0.01 \mathrm{~m}$. The beam material has Young's modulus of $1.2 \times 10^{11} \mathrm{~Pa}$ and Poison's ratio of 0 so that the bending stiffness reads $E I=10^{2} \mathrm{Nm}^{2}$. The two loads $M=200 \pi \lambda$ and $F=50 \lambda$ increase simultaneously and incrementally from $\lambda=0$ to $\lambda=1$ in 1000 steps. The beam is modeled via 100 solid elements of ALE-ANCF in the length direction with all the material coordinates fixed. 


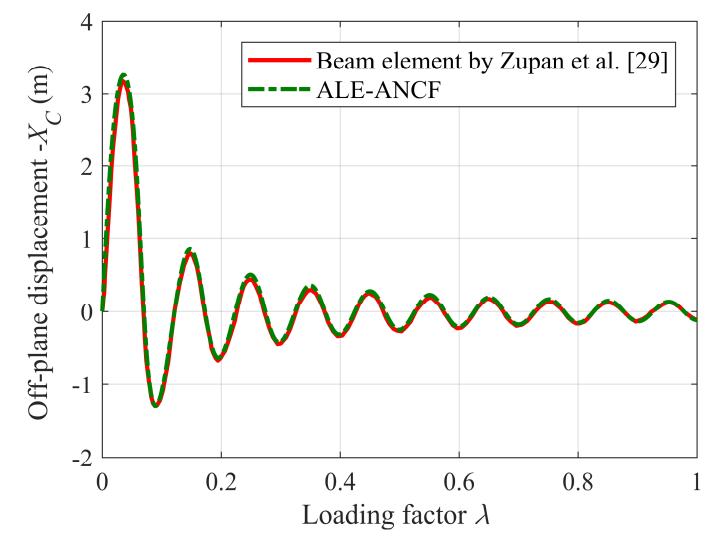

Fig. 5 Off-plane displacement $-X_{C}$ of free end centroid $C$ versus loading factor $\lambda$

Fig. 4 illustrates the configurations of the beam under different loading factors. When $\lambda=1$, the beam bends into a helical curve with 10 circles and is in the opposite direction of the concentrated force $F$, as shown in Fig. 4 (d). Fig. 4(d) also indicates that for the final configuration, i.e., $\lambda=1$, the free end cross-section of the beam has a torsional angle of about $0.2556 \pi$. Fig. 4 shows that at different loading cases, the beam bends to different sides of YZ-plane. This phenomenon can also be observed in Fig. 5, which presents the off-plane displacement of the free end centroid $C$ with an increase of the loading factor $\lambda$. In Fig. 5, the red solid curve represents the results modeled via the geometrically exact three-dimensional beam which uses the quaternions to describe the rotations by Zupan et al. [29], the green dash-dotted curve denotes the results modeled via the solid elements of ALE-ANCF in this study. For a good comparison, the off-plane displacement in the present study is computed as the negative $X$-coordinate of point $C$. Fig. 5 shows a good agreement between the results via ALE-ANCF model and the results by Zupan et al. [29].

\subsection{Dynamics of a flexible pendulum}

The second example is a dynamic problem studied by Olshevskiy et al. [2, 6] in order to validate different kinds of solid elements of ANCF undergoing both large overall motions and large deformations. In this study, this dynamic example is utilized to validate the accuracy and convergence of the variable-length solid element of ALE-ANCF in comparison with the traditional solid element of ANCF $[1,6]$.

As shown in Fig. 6, the flexible pendulum is a short beam with a length of $0.5 \mathrm{~m}$ and a square cross-section of $0.1 \mathrm{~m} \times 0.1 \mathrm{~m}$. The mass density, Young's modulus and Poison's ratio of the pendulum are $7800 \mathrm{~kg} / \mathrm{m}^{3}, 2 \times 10^{6} \mathrm{~Pa}$ and 0.3 , respectively. The pendulum is initially in the vertical position in Fig. 6 , where two nodes of the short horizontal edge of the pendulum are constrained to the ground via two revolute joints, and then starts falling down due to gravity. For the motion of the pendulum, the vector Q of external forces in Eq. (26) comes from gravity and damping, and the vector of corresponding damping force yields [6]

$$
\mathbf{Q}=-\mathbf{M} \dot{\mathbf{q}}_{D} .
$$




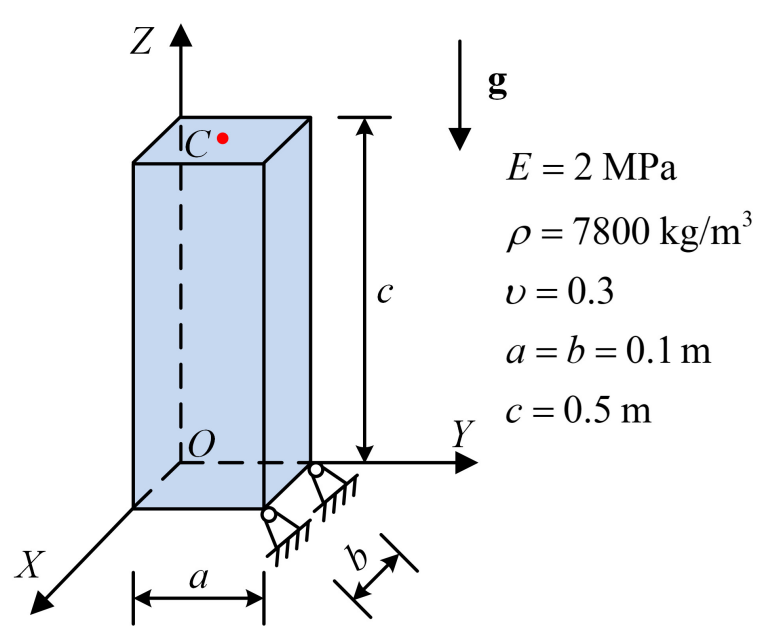

Fig. 6 A schematic diagram of a flexible pendulum

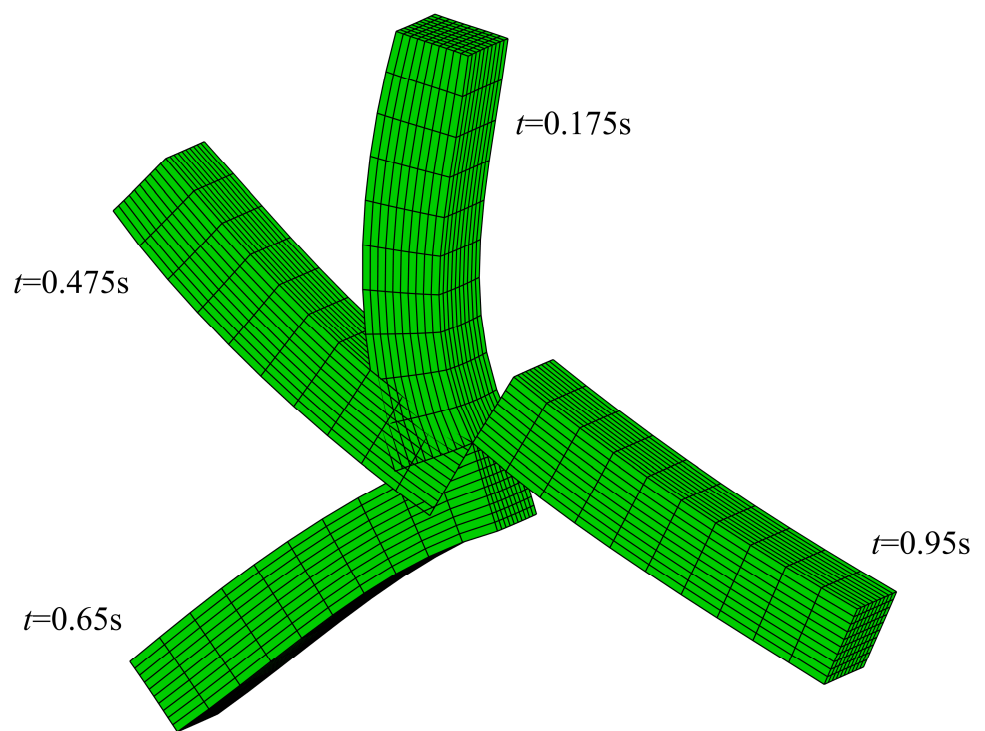

Fig. 7 Configurations of the flexible pendulum modeled via one solid element of ALE-ANCF

Fig. 7 shows the deformed shapes of the flexible pendulum at four moments modeled via one solid element of ALE-ANCF. For visualization purpose, each solid element of ALE-ANCF is plotted via a $10 \times 10 \times 10$ mesh in Fig. 7. As the length of the pendulum is constant, the velocities of the material coordinates are equal to zeros, that is, $\dot{h}_{1}=\dot{h}_{2}=0$. Fig. 8 presents the energy balance of the flexible pendulum modeled via one solid element of ALE-ANCF with respect to time when the damping force in Eq. (27) is neglected, and shows that the total energy keeps constant as the only external force comes from gravity. It also implies that the generalized- $\alpha$ method [23-25] is able to conserve energy when solving the DAEs in Eq. (26). 


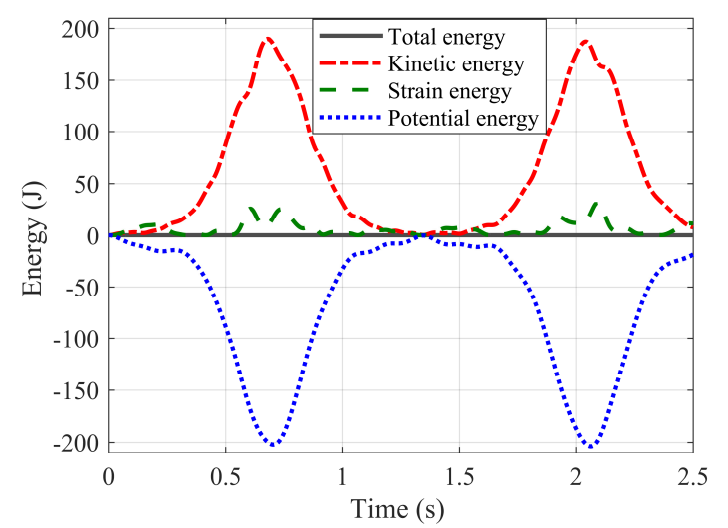

Fig. 8 Energy balance of the undamped flexible pendulum with respect to time
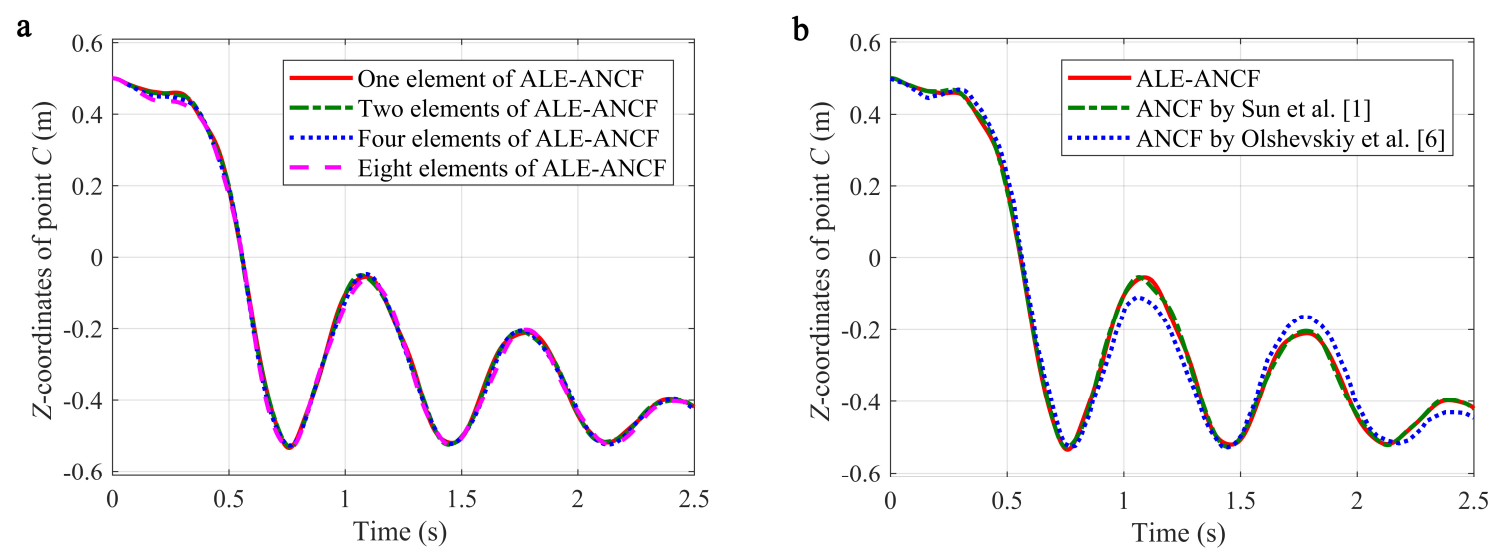

Fig. 9 Comparisons of Z-coordinates of point $C$. a Different numbers of solid elements. b ALE-ANCF and ANCF models

To illustrate the convergence of the solid element of ALE-ANCF, the pendulum is modeled via different numbers of elements. Fig. 9(a) illustrates the Z-coordinate responses of the centroid $C$ of the free-end cross-section modeled by 1, 2, 4 and 8 solid elements, respectively and shows a good agreement among them. Fig. 9(b) gives the comparisons of $Z$-coordinate responses of point $C$ for the three different models. The red solid curve is the result modeled by one solid element of ALE-ANCF proposed in this study, the green dash-dotted curve represents the result modeled by one solid element of ANCF derived by Sun et al. [1], and the blue short dashed curve denotes the result modeled by one solid element of ANCF derived by Olshevskiy et al. [6]. Fig. 9(b) shows that the result from ALE-ANCF model matches with ANCF model by Sun et al. [1] very well, and is similar to that by Olshevskiy et al. [6]. The little difference in Fig. 9(b) probably comes from that the formulae of the elastic force of the solid element of ANCF are not given by Olshevskiy et al. [6].

\subsection{Dynamics of an axially moving beam with variable length}

This is a classic dynamic problem studied by many researchers [30-32] to validate different axially moving beam models. To validate the variable-length characteristic of the solid element of ALE-ANCF in this study, this example is 
reanalyzed and modeled by the solid element of ALE-ANCF. The results are compared with those by Park et al. [30].

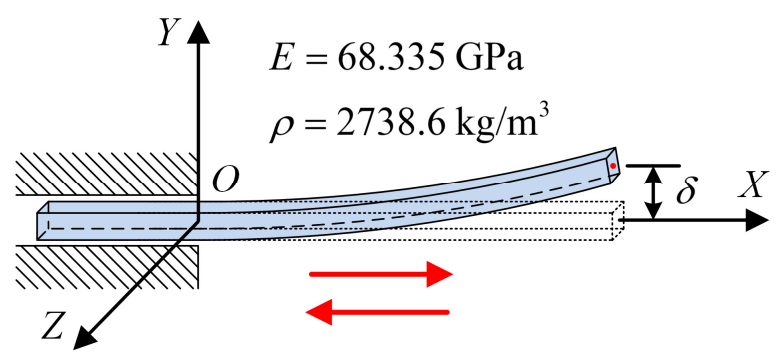

Fig. 10 A schematic diagram of an axially moving beam

As shown in Fig. 10, a flexible beam moves axially from a rigid hub fixed in space. The beam has the same geometric and material parameters as those in [30]. That is, the total length of the beam is $3.657 \mathrm{~m}$, the cross-sectional area and the second moment of the cross-section area are $1.4661 \times 10^{-3} \mathrm{~m}^{2}$ and $1.1073 \times 10^{-8} \mathrm{~m}^{4}$, respectively. The density, Young's modulus and Poison's ratio of the beam material are $2738.6 \mathrm{~kg} / \mathrm{m}^{3}, 6.8335 \times 10^{10} \mathrm{~Pa}$ and 0.3 , respectively. In this study, the axially moving beam is modeled via the variable-length solid element of ALE-ANCF and studied via two cases.

The first case of concern is the deployment of the beam at a constant moving velocity of $0.1 \mathrm{~m} / \mathrm{s}$. The beam having an initial length of $1.8 \mathrm{~m}$ outside the rigid hub is modeled via 3 solid elements of ALE-ANCF in the length direction. During the deployment, the maximum length of the boundary element is set as $0.9 \mathrm{~m}$, which means four nodes of ALE-ANCF are inserted to divide the boundary element into two elements when the length of boundary element reaches $0.9 \mathrm{~m}$, as shown in Fig. 2(a). To obtain the transverse displacement response, the free end of the beam has an initial static deflection $\delta_{0}=5 \mathrm{~mm}$. Fig. 11(a) illustrates the comparison results of the transverse displacement $\delta$ between the ALE-ANCF model and the axially moving beam model by Park et al. [30]. From Fig. 11 (a), it can be found that the results from the ALE-ANCF model agree with those by Park et al. [30] very well, except for those around $t=3 \mathrm{~s}$ and $t=9 \mathrm{~s}$. This is probably due to the reason that the inserted nodes of ALE-ANCF may have an impact on the configuration of the beam, and as a result, on the dynamic response. 

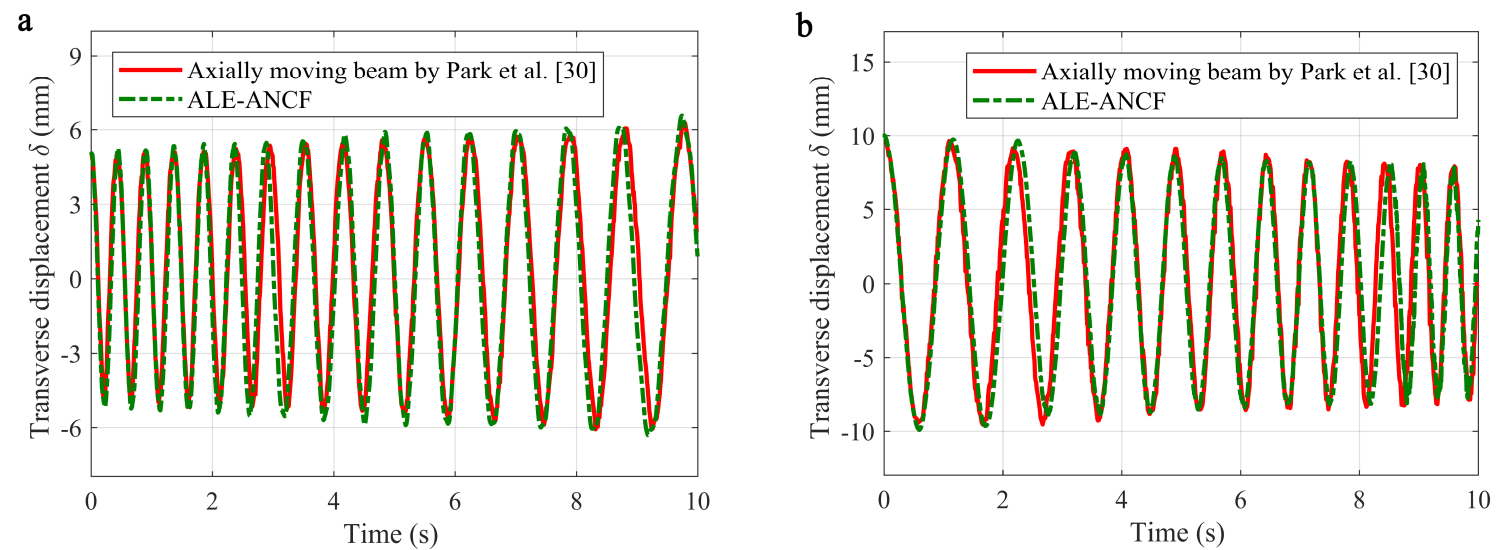

Fig. 11 Comparisons of the transverse displacements of the free end of the beam. a Deployment of the beam. b Retraction of the beam

The second case is the retraction of the beam at a constant moving velocity of $0.1 \mathrm{~m} / \mathrm{s}$. The beam with an initial length of $3 \mathrm{~m}$ outside the rigid hub is modeled via 5 solid elements of ALE-ANCF in the length direction. During the retraction, the minimum length of the boundary element is set as $0.3 \mathrm{~m}$. This means that when the length of the boundary element decreases to $0.3 \mathrm{~m}$, the two boundary elements will merge into a single element, as shown in Fig. 2 (b). The initial static deflection of the free end of the beam is set as $\delta_{0}=10 \mathrm{~mm}$ for this case. Fig. 11(b) shows that the results from the ALE-ANCF model have a good agreement with those by Park et al. [30]. The impacts of the deleted nodes of ALE-ANCF on the dynamic response can also be observed around $t=3 \mathrm{~s}$ and $t=9 \mathrm{~s}$. From Fig. 11(a) and Fig. 11(b), it can be concluded that the impacts of the inserted and deleted nodes of ALE-ANCF on the dynamic response are acceptable. The impacts can be reduced by an appropriate selection of the maximum and minimum lengths of the boundary element.

\section{Conclusions}

The paper presents an axially variable-length solid element with eight nodes of the ALE-ANCF. The modeling scheme enables one to accurately describe the dynamics of a 3D FMBS with variable-length bodies undergoing both large overall motion and large deformation. The paper also presents the formulae of the elastic forces and the additional inertial forces, as well as the corresponding Jacobian formulations of the solid element of ALE-ANCF. The paper gives the accuracy and convergence validations of the solid element via one static and two dynamic benchmark examples, and the comparison among the variable-length solid element of ALE-ANCF, the conventional solid element of ANCF and the axially moving beam element. The results reveal that the axially variable-length solid element of ALE-ANCF proposed in the paper is accurate. The solid element makes it possible to simulate the dynamics multibody systems in engineering and to optimize 3D variable-length flexible bodies of those multibody systems, such as an axially moving beam carrying a tip mass and the first-stage deployment of a spinning solar sail [21]. 
Acknowledgements This work was supported in part by the National Natural Science Foundation of China under Grants 11521062 and 11722216 and by the 111 China Project (B16003). It was also supported in part by Postgraduate Research \& Practice Innovation Program of Jiangsu Province under Grants KYCX17_0226 and by China Scholarship Council.

\section{Appendix}

The detailed expressions of the invariant matrices in Eq. (14) are given as follows

$$
\begin{aligned}
& \left(\mathbf{K}_{1}\right)_{k m n i}=\int_{V_{0}}(\mathbf{E})_{11}\left((\mathbf{A})_{k m}(\mathbf{A})_{n i}+(\mathbf{B})_{k m}(\mathbf{B})_{n i}+(\mathbf{C})_{k m}(\mathbf{C})_{n i}\right) \mathrm{d} V, \\
& \left(\mathbf{K}_{2}\right)_{k m n i}=\int_{V_{0}}(\mathbf{E})_{12}\left((\mathbf{A})_{k m}(\mathbf{B})_{n i}+(\mathbf{A})_{k m}(\mathbf{C})_{n i}+(\mathbf{B})_{k m}(\mathbf{A})_{n i}\right. \\
& \left.+(\mathbf{B})_{k m}(\mathbf{C})_{n i}+(\mathbf{C})_{k m}(\mathbf{A})_{n i}+(\mathbf{C})_{k m}(\mathbf{B})_{n i}\right) \mathrm{d} V \\
& \left(\mathbf{K}_{3}\right)_{k m n i}=\int_{V_{0}} 2(\mathbf{E})_{44}\left((\mathbf{D})_{k m}(\mathbf{D})_{n i}+(\mathbf{D})_{k m}(\mathbf{D})_{i n}+(\mathbf{G})_{k m}(\mathbf{G})_{n i}\right. \\
& \left.+(\mathbf{G})_{k m}(\mathbf{G})_{i n}+(\mathbf{H})_{k m}(\mathbf{H})_{n i}+(\mathbf{H})_{k m}(\mathbf{H})_{i n}\right) \mathrm{d} V, \\
& \left(\mathbf{K}_{4}\right)_{n i}=\int_{V_{0}}(\mathbf{E})_{11}\left((\mathbf{A})_{n i}+(\mathbf{B})_{n i}+(\mathbf{C})_{n i}\right) \mathrm{d} V \text {, } \\
& \left(\mathbf{K}_{5}\right)_{n i}=\int_{V_{0}} 2(\mathbf{E})_{12}\left((\mathbf{A})_{n i}+(\mathbf{B})_{n i}+(\mathbf{C})_{n i}\right) \mathrm{d} V, \\
& \left(\mathbf{K}_{6}\right)_{k m n l i}=\int_{V_{0}}(\mathbf{E})_{11}\left((\mathbf{A})_{k m}(\mathbf{A})_{n l, q_{i}}+(\mathbf{B})_{k m}(\mathbf{B})_{n l, q_{i}}+(\mathbf{C})_{k m}(\mathbf{C})_{n l, q_{i}}\right) \mathrm{d} V, \\
& \left(\mathbf{K}_{7}\right)_{k m n l i}=\int_{V_{0}}(\mathbf{E})_{12}\left((\mathbf{A})_{k m}(\mathbf{B})_{n l, q_{i}}+(\mathbf{A})_{k m}(\mathbf{C})_{n l, q_{i}}+(\mathbf{B})_{k m}(\mathbf{A})_{n l, q_{i}}\right. \\
& \left.+(\mathbf{B})_{k m}(\mathbf{C})_{n l, q_{i}}+(\mathbf{C})_{k m}(\mathbf{A})_{n l, q_{i}}+(\mathbf{C})_{k m}(\mathbf{B})_{n l, q_{i}}\right) \mathrm{d} V \\
& \left(\mathbf{K}_{8}\right)_{k m n l i}=\int_{V_{0}} 4(\mathbf{E})_{44}\left((\mathbf{D})_{k m}(\mathbf{D})_{n l, q_{i}}+(\mathbf{G})_{k m}(\mathbf{G})_{n l, q_{i}}+(\mathbf{H})_{k m}(\mathbf{H})_{n l, q_{i}}\right) \mathrm{d} V \text {, } \\
& \left(\mathbf{K}_{9}\right)_{n l i}=\int_{V_{0}}(\mathbf{E})_{11}\left((\mathbf{A})_{n l, q_{i}}+(\mathbf{B})_{n l, q_{i}}+(\mathbf{C})_{n l, q_{i}}\right) \mathrm{d} V \text {, } \\
& \left(\mathbf{K}_{10}\right)_{n l i}=\int_{V_{0}} 2(\mathbf{E})_{12}\left((\mathbf{A})_{n l, q_{i}}+(\mathbf{B})_{n l, q_{i}}+(\mathbf{C})_{n l, q_{i}}\right) \mathrm{d} V,
\end{aligned}
$$

where the subscripts $k, m, n, l=1,2, \cdots, 96$ and $i=1,2, \cdots, 98$. From Eq. (A.1), it can be observed that $\mathbf{K}_{1}$ and $\mathbf{K}_{2}$ are symmetric about $k, m$, and $n$, $i$, respectively. $\mathbf{K}_{3}$, $\mathbf{K}_{4}$ and $\mathbf{K}_{5}$ are symmetric about $n, i . \mathbf{K}_{6}$ and $\mathbf{K}_{7}$ are symmetric about $k, m$, and $n$, l, respectively. $\mathbf{K}_{8}, \mathbf{K}_{9}$ and $\mathbf{K}_{10}$ are symmetric about $n, l$.

The detailed expressions of the invariant matrices in Eq. (20) are given as follows

$$
\begin{aligned}
& \left(\mathbf{K}_{11}\right)_{i k}=2 \rho \int_{V_{0}}\left(\mathbf{N}_{e}\right)_{d i}\left(\mathbf{N}_{e, h_{1}}\right)_{d k} \mathrm{~d} V, \\
& \left(\mathbf{K}_{12}\right)_{i k}=2 \rho \int_{V_{0}}\left(\mathbf{N}_{e}\right)_{d i}\left(\mathbf{N}_{e, h_{2}}\right)_{d k} \mathrm{~d} V,
\end{aligned}
$$




$$
\begin{aligned}
& \left(\mathbf{K}_{13}\right)_{i k}=\rho \int_{V_{0}}\left(\mathbf{N}_{e}\right)_{d i}\left(\mathbf{N}_{e, h_{1} h_{1}}\right)_{d k} \mathrm{~d} V, \\
& \left(\mathbf{K}_{14}\right)_{i k}=\rho \int_{V_{0}}\left(\mathbf{N}_{e}\right)_{d i}\left(\mathbf{N}_{e, h_{2} h_{2}}\right)_{d k} \mathrm{~d} V, \\
& \left(\mathbf{K}_{15}\right)_{i k}=2 \rho \int_{V_{0}}\left(\mathbf{N}_{e}\right)_{d i}\left(\mathbf{N}_{e, h_{1} h_{2}}\right)_{d k} \mathrm{~d} V, \\
& \left(\mathbf{K}_{16}\right)_{i l k}=2 \rho \int_{V_{0}}\left(\mathbf{N}_{e, q_{i}}\right)_{d l}\left(\mathbf{N}_{e, h_{1}}\right)_{d k} \mathrm{~d} V, \\
& \left(\mathbf{K}_{17}\right)_{i l k}=2 \rho \int_{V_{0}}\left(\mathbf{N}_{e, q_{i}}\right)_{d l}\left(\mathbf{N}_{e, h_{2}}\right)_{d k} \mathrm{~d} V, \\
& \left(\mathbf{K}_{18}\right)_{i l k}=\rho \int_{V_{0}}\left(\mathbf{N}_{e, q_{i}}\right)_{d l}\left(\mathbf{N}_{e, h_{1} h_{1}}\right)_{d k} \mathrm{~d} V, \\
& \left(\mathbf{K}_{19}\right)_{i l k}=\rho \int_{V_{0}}\left(\mathbf{N}_{e, q_{i}}\right)_{d l}\left(\mathbf{N}_{e, h_{2} h_{2}}\right)_{d k} \mathrm{~d} V, \\
& \left(\mathbf{K}_{20}\right)_{i l k}=2 \rho \int_{V_{0}}\left(\mathbf{N}_{e, q_{i}}\right)_{d l}\left(\mathbf{N}_{e, h_{1} h_{2}}\right)_{d k} \mathrm{~d} V,
\end{aligned}
$$

where the subscripts $k, l=1,2, \cdots, 96, d=1,2,3$, and $i=1,2, \cdots, 98$.

The matrices in Eqs. (A.1) and (A.2) are all time-invariant and can be computed and stored by using sparse matrix technique in the preprocessing procedure to greatly improve the computation efficiency.

\section{References}

1. Sun, J.L., Tian, Q., Hu, H.Y.: Topology optimization of a three-dimensional flexible multibody system via moving morphable components. Journal of Computational and Nonlinear Dynamics. 13(2), 021010 (2018)

2. Olshevskiy, A., Dmitrochenko, O., Yang, H., Kim, C.: Absolute nodal coordinate formulation of tetrahedral solid element. Nonlinear Dynamics. 88(4), 2457-2471 (2017)

3. Gerstmayr, J., Sugiyama, H., Mikkola, A.: Review on the absolute nodal coordinate formulation for large deformation analysis of multibody systems. Journal of Computational and Nonlinear Dynamics. 8(3), 031016 (2013)

4. Hu, H.Y., Tian, Q., Liu, C.: Computational dynamics of soft machines. Acta Mechanica Sinica. 33(3), 516-528 (2017)

5. Shabana, A.A.: An absolute nodal coordinates formulation for the large rotation and deformation analysis of flexible bodies. Report, No. MBS96-1-UIC, University of Illinois at Chicago (1996)

6. Olshevskiy, A., Dmitrochenko, O., Kim, C.: Three-dimensional solid brick element using slopes in the absolute nodal coordinate formulation. Journal of Computational and Nonlinear Dynamics. 9, 021001 (2014)

7. Wei, C., Wang, L., Shabana, A.A.: A total Lagrangian ANCF liquid sloshing approach for multibody system applications. Journal of Computational and Nonlinear Dynamics. 10(5), 051014 (2015)

8. Pappalardo, C.M., Wang, T., Shabana, A.A.: Development of ANCF tetrahedral finite elements for the nonlinear dynamics of flexible structures. Nonlinear Dynamics. 89(4), 
2905-2932 (2017)

9. Tang, J.L., Ren, G.X., Zhu, W.D., Ren, H.: Dynamics of variable-length tethers with application to tethered satellite deployment. Communications in Nonlinear Science and Numerical Simulation. 16(8), 3411-3424 (2011)

10. Escalona, J.L.: An arbitrary Lagrangian-Eulerian discretization method for modeling and simulation of reeving systems in multibody dynamics. Mechanism and Machine Theory. 112, 1-21 (2017)

11. Du, J.L., Cui, C.Z., Bao, H., Qiu, Y.Y.: Dynamic analysis of cable-driven parallel manipulators using a variable length finite element. Journal of Computational and Nonlinear Dynamics. 10(1), 011013 (2015)

12. Gross, D., Messner, D.: The able deployable articulated mast-enabling technology for the shuttle radar topography mission. In: Proceedings of the 33rd Aerospace Mechanisms Symposium, Pasadena, California, May 19-21, (1999)

13. Hayashi, H., Takehara, S., Terumichi, Y.: Numerical approach for flexible body motion with large displacement and time-varying length. The 3rd Joint International Conference on Multibody System Dynamics and the 7th Asian Conference on Multibody Dynamics, BEXCO, Busan, Korea, June 30-July 3 (2014)

14. Terumichi, Y., Kaczmarczyk, S., Sogabe, K.: Numerical approach in the analysis of flexible body motion with time-varying length and large displacement using multiple time scales. The 1st Joint International Conference on Multibody System Dynamics, Lappeenranta, Finland, May 25-27 (2010)

15. Hong, D.F., Ren, G.X.: A modeling of sliding joint on one-dimensional flexible medium. Multibody System Dynamics. 26(1), 91-106 (2011)

16. Hyldahl, P., Mikkola, A., Balling, O.: A thin plate element based on the combined arbitrary Lagrange-Euler and absolute nodal coordinate formulations. Proceedings of the Institution of Mechanical Engineers, Part K: Journal of Multi-Body Dynamics. 227(3), 211-219 (2013)

17. Yang, S., Deng, Z.Q., Sun, J., Zhao, Y., Jiang, S.Y.: A variable-length beam element incorporating the effect of spinning. Latin American Journal of Solids and Structures. 14, 1506-1528 (2017)

18. Hong, D.F., Tang, J.L., Ren, G.X.: Dynamic modeling of mass-flowing linear medium with large amplitude displacement and rotation. Journal of Fluids and Structures. 27(8), 1137-1148 (2011)

19. Shabana, A.A.: Definition of ANCF finite elements. Journal of Computational and Nonlinear Dynamics. 10(5), 054506 (2015)

20. Sun, J.L., Tian, Q., Hu, H.Y., Pedersen, N.L.: Topology optimization of a flexible multibody system with variable-length bodies described by ALE-ANCF. Nonlinear Dynamics. 93(2), 413-441 (2018)

21. Sun, J.L., Tian, Q., Hu, H.Y., Pedersen, N.L.: Simultaneous topology and size optimization of a 3D variable-length structure described by the ALE-ANCF. Mechanism and Machine Theory. 129, 80-105 (2018)

22. Goldstein, H., Poole, C., Safko, J.: Classical mechanics. Pearson Education, Inc., 16-21 2002.

23. Brüls, O., Arnold, M.: The generalized- $\alpha$ scheme as a linear multistep integrator: 
Toward a general mechatronic simulator. Journal of Computational and Nonlinear Dynamics. 3 (2008)

24. Tian, Q., Flores, P., Lankarani, H.M.: A comprehensive survey of the analytical, numerical and experimental methodologies for dynamics of multibody mechanical systems with clearance or imperfect joints. Mechanism and Machine Theory. 122, 1-57 (2018)

25. Chung, J., Hulbert, G.M.: A time integration algorithm for structural dynamics with improved numerical dissipation: The generalized $\alpha$-method. Journal of Applied Mechanics. 60(2), 371-375 (1993)

26. Ibrahimbegovic, A.: On the choice of finite rotation parameters. Computer Methods in Applied Mechanics and Engineering. 149(1-4), 49-71 (1997)

27. Zupan, D., Saje, M.: Finite-element formulation of geometrically exact three-dimensional beam theories based on interpolation of strain measures. Computer Methods in Applied Mechanics and Engineering. 192(49-50), 5209-5248 (2003)

28. Zhang, R., Zhong, H.: Weak form quadrature element analysis of spatial geometrically exact shear-rigid beams. Finite Elements in Analysis and Design. 87, 22-31 (2014)

29. Zupan, E., Saje, M., Zupan, D.: The quaternion-based three-dimensional beam theory. Computer Methods in Applied Mechanics and Engineering. 198(49-52), 3944-3956 (2009)

30. Park, S., Hong, H.Y., Chung, J.: Vibrations of an axially moving beam with deployment or retraction. AIAA Journal. 51(3), 686-696 (2013)

31. Chang, J., Lin, W., Huang, C., Choi, S.: Vibration and stability of an axially moving Rayleigh beam. Applied Mathematical Modelling. 34(6), 1482-1497 (2010)

32. Al-Bedoor, B.O., Khulief, Y.A.: Vibrational motion of an elastic beam with prismatic and revolute joints. Journal of Sound and Vibration. 190(2), 195-206 (1996) 